\title{
Femtosecond laser written waveguides deep inside silicon
}

\author{
I. Pavlov, ${ }^{1}$ O. Tokel, ${ }^{1}$ (i) S. Pavlova, ${ }^{2}$ V. Kadan, ${ }^{2}$ G. Makey, ${ }^{1}$ A. Turnali, ${ }^{3}$ \\ Ö. YAVUZ, ${ }^{3}$ AND F. Ö. ILDAY ${ }^{1,3, \star}$ (1) \\ ${ }^{1}$ Department of Physics, Bilkent University, Ankara 06800, Turkey \\ ${ }^{2}$ Institute of Physics, National Academy of Sciences of Ukraine, Kiev 03028, Ukraine \\ ${ }^{3}$ Department of Electrical and Electronics Engineering, Bilkent University, Ankara 06800, Turkey \\ ${ }^{4}$ UNAM - National Nanotechnology Research Center and Institute of Materials Science and Nanotechnology, Bilkent University, Ankara 06800, Turkey \\ *Corresponding author: ilday@bilkent.edu.tr
}

Received 28 April 2017; revised 15 June 2017; accepted 16 June 2017; posted 16 June 2017 (Doc. ID 294915); published 31 July 2017

Photonic devices that can guide, transfer, or modulate light are highly desired in electronics and integrated silicon (Si) photonics. Here, we demonstrate for the first time, to the best of our knowledge, the creation of optical waveguides deep inside $\mathrm{Si}$ using femtosecond pulses at a central wavelength of $1.5 \mu \mathrm{m}$. To this end, we use $350 \mathrm{fs}$ long, $2 \mu \mathrm{J}$ pulses with a repetition rate of $250 \mathrm{kHz}$ from an Er-doped fiber laser, which we focused inside $S i$ to create permanent modifications of the crystal. The position of the beam is accurately controlled with pump-probe imaging during fabrication. Waveguides that were $5.5 \mathrm{~mm}$ in length and $20 \mu \mathrm{m}$ in diameter were created by scanning the focal position along the beam propagation axis. The fabricated waveguides were characterized with a continuous-wave laser operating at $1.5 \mu \mathrm{m}$. The refractive index change inside the waveguide was measured with optical shadowgraphy, yielding a value of $6 \times 10^{-4}$, and by direct light coupling and far-field imaging, yielding a value of $3.5 \times 10^{-4}$. The formation mechanism of the modification is discussed. () 2017 Optical Society of America

OCIS codes: (230.7370) Waveguides; (140.7090) Ultrafast lasers; (140.3390) Laser materials processing; (130.0130) Integrated optics.

https://doi.org/10.1364/OL.42.003028

Integration of optics with silicon ( $\mathrm{Si}$ )-based computing devices has been investigated since the 1960s [1]. Earlier work focused on optical logic; however, the greatest benefit of optical integration is expected to come from optical interconnects operating with low delay times and low power dissipation [2]. Such optical data transfer links are progressively finding use at shorter distances, providing advantages from high transmission rates enabled by wavelength division multiplexed operation over waveguides [3]. However, a major limitation in applying optical interconnects at short distances for chip-to-chip or potentially intra-chip connections is due to challenges in creating dense optical interconnects [4]. For instance, optical waveguide architectures positioned over chip surfaces suffer from signal crosstalk between intersecting channels [5]. Novel interconnect architectures such as multi-layered waveguides are proposed as a solution to some of these problems, help with interconnect scaling, and complement electrical connections suffering from lower bandwidths [6]. Toward these goals, variants of threedimensional (3D) laser micro-fabrication approaches have been explored in the past decade in various materials [7]. Through the nonlinear processes taking place during ultrafast laser material interaction $[8,9]$, laser light can impart a permanent refractive index change in the volume of transparent materials, thus enabling the fabrication of waveguides in glasses, polymers, lithium niobate, and other crystals [10-15], with important applications in integrated optics $[12,16]$ and, recently, quantum circuits [17]. However, despite the importance of $\mathrm{Si}$ for the micro-electronics industry and the growing importance of Si photonics, functional waveguides with simple geometries deep inside $\mathrm{Si}$ have not been shown. One of the previous attempts, where a $2.4-\mu \mathrm{m}$ femtosecond laser was used for direct writing of waveguides in $\mathrm{Si}$, allowed the creation of a waveguide only in the close vicinity of the front surface [18], thus limiting applications to two-dimensional geometry.

The lack of functional waveguides or, in general, in-chip devices is due to difficulties in $3 \mathrm{D}$ laser processing of $\mathrm{Si}$ without altering the wafer surface $[19,20]$. We have first shown the possibility of laser processing deep inside Si using nanosecond pulses at $1.55 \mu \mathrm{m}$ [21], where $\mathrm{Si}$ is transparent. This approach has been subsequently developed into a comprehensive technique that enables creation of arbitrary complex 3D microstructures inside Si with $1-\mu \mathrm{m}$ resolution [22]. The laserinduced refractive index changes are used to realize functional elements inside $\mathrm{Si}$, in-chip photonic structures and devices, including lenses, gratings, phase-type, high-resolution holograms, as well as waveguides [22]. However, the laser-induced index change is negative with nanosecond pulses, which requires tubular or similar waveguide structures. Using a technique based on the one we have demonstrated in Ref. [22], the possible creation of waveguides using nanosecond pulses have recently been reported [23]. However, no experimental evidence of actual guidance of light was provided, only that the scattered 
light followed a linear pattern. Given that nanosecond pulses produce negative changes in the refractive index based on our results with similar pulse durations and energies, it is not clear if the reported structures indeed were functional as waveguides.

In this Letter, we extend the subsurface Si modification capability to femtosecond lasers. To the best of our knowledge, this is the first report of $\mathrm{Si}$ subsurface modification with a femtosecond laser without altering the wafer surface. We overcome previously reported difficulties in direct-laser processing of $\mathrm{Si}$ below the surface $[19,20]$ by benefiting from cumulative effects arising from the use of high repetition rates $(250 \mathrm{kHz})$, in a manner that is loosely analogous to [24]. We furthermore provide clear evidence that the structures are indeed waveguiding, tested at $1.5 \mu \mathrm{m}$ telecommunication wavelength. The structures are created while the laser focus is monitored with in situ pump-probe imaging. The laser-written modifications are then used to fabricate buried waveguides with a cylindrically symmetric geometry. The refractive index profiles of the waveguides are characterized with quantitative shadowgraphy, revealing that they are approximately pure phase objects, making them ideal for low-loss operation.

The laser processing setup with pump-probe imaging is shown in Fig. 1(a). The laser source is a home-built Er-doped fiber laser, which can emit pulses of more than $2 \mu \mathrm{J}$ energy with a $350 \mathrm{fs}$ pulse width (full width at half-maximum). The repetition rate of the laser is tunable from $1 \mathrm{MHz}$ to $100 \mathrm{kHz}$, but was set to $250 \mathrm{kHz}$ in this Letter. The laser output was split into two parts (pump and probe) by the combination of a half-wave plate (HWP) and a polarization-beam splitter (PBS), which allows us to continuously tune the power ratio between the two arms. A second pair of HWPs and PBSs allows independent

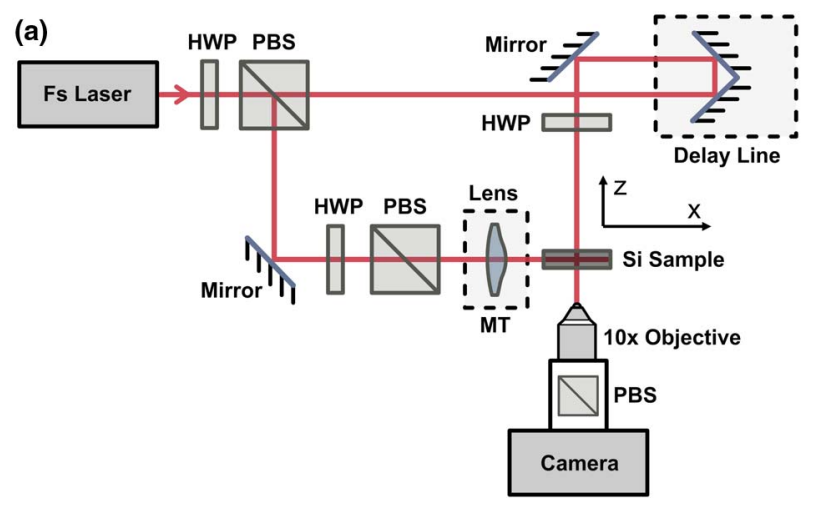

(b)

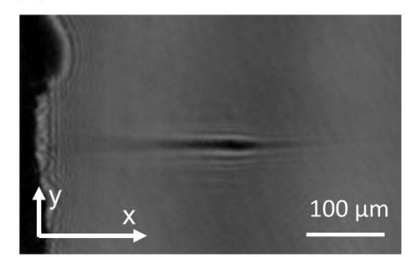

(c)

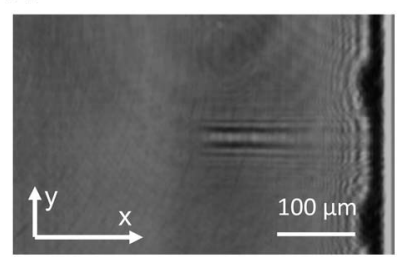

Fig. 1. (a) Experimental setup used for pump-probe imaging and for fabricating subsurface waveguides inside Si. (b) Pump-probe image of laser-induced plasma in $\mathrm{Si}$, obtained with a 10 ps delay between the pump and probe, indicating that the focal position of the beam is $\sim 200 \mu \mathrm{m}$ below the front surface. (c) Permanently modified area near the back surface of the sample, recorded after the pump beam is turned off. HWP, half-wave plate; PBS, polarization beam splitter; MT, motorized stage; FS Laser, femtosecond laser. The pump beam is propagating along the direction of the $\mathrm{x}$-axis. control of power on the pump arm, delivering a vertically polarized (y-axis in Fig. 1) pump beam to the sample, which is necessary for time-resolved imaging of the ultrafast laser pulse interaction with transparent materials [25]. The pump beam is focused in $\mathrm{Si}$ with a 0.5 numerical aperture (NA) aspheric lens (Thorlabs C240TME-C), mounted on a translation stage. The stage allows us to translate the focal spot of the pump beam along the propagation direction during waveguide fabrication. The diameter of the pump beam in front of the lens was $6 \mathrm{~mm}$ at a $1 / e^{2}$ level of maximum intensity. For the probe arm, a retro-reflector mirror was implemented, which was mounted on a motorized stage, in order to adjust the delay time between the pump and probe pulses. An additional pair of HWPs and PBSs is placed into the probe arm before and after the Si sample, which allows for pump-probe imaging in both parallel and cross-polarizations using an InGaAs camera equipped with a $10 \times$ objective. The sample position is controlled with a $3 \mathrm{D}$ motorized translation stage (not shown in the figure). In this Letter, we used $1 \mathrm{~mm}$ thick, double-side-polished, $\langle 100\rangle$-cut, p-type Si samples (boron doped with resistivity of $1 \Omega \cdot \mathrm{cm}$ ). The characterization of the waveguides was done with an additional low-power, continuous-wave (cw) laser, operating at $1.5 \mu \mathrm{m}$, coupled to the same setup in place of the pump arm.

A time-resolved pump-probe image of a femtosecond pulse interacting with $\mathrm{Si}$ is shown in Fig. 1(b). The dark shadow of the recorded image is based on free carrier absorption (FCA) induced by the pump pulse. We note that FCA is a transient effect, disappearing after a time equal to the free carrier lifetime in $\mathrm{Si}$, which is more than $10 \mathrm{~ns}$. The time delay between the pump and probe can be changed over a wide range, up to a few nanoseconds, to acquire information on the plasma. A more detailed analysis of the transient dynamics of ultrashort pulse propagation in $\mathrm{Si}$ will be presented in a future publication. If we set the time delay between the pump and probe arms much more then the propagation time of the pump pulse through the sample [10 ps for Fig. 1(b)], while keeping it less then the free carrier relaxation time, we can visualize the position of the sample where the pump pulse intensity was maximum, i.e., the focal position of the pump. In this Letter, we used this capability to directly identify the pump beam behavior in $\mathrm{Si}$, which was crucial to both locating the focused beam and to controllably writing subsurface waveguides.

In our experiments, we found that a pump beam of $\sim 2 \mu \mathrm{J}$ pulse energy at a repetition rate of $250 \mathrm{kHz}$ focused in Si produces permanent changes in the sample [Fig. 1(c)]. Controlled subsurface modifications are realized by focusing the pump beam on or close to a previously modified subsurface section. Each modified section acts as a seed for the next part, if one simply pulls the structures along a linear geometry, either by translating the sample or focusing lens. By translating the focal position of the pump beam from the back to the front surface [Figs. 1(b)-1(c)] parallel to beam propagation direction, we created $\sim 5 \mathrm{~mm}$ long, cylindrically symmetric wire-like structures with $\sim 20 \mu \mathrm{m}$ diameter. We found that the optimal scanning speeds were in the range of $0.03-0.1 \mathrm{~mm} / \mathrm{s}$. The presence of an upper limit on the scanning speed $(0.1 \mathrm{~mm} / \mathrm{s}$ at the given pulse energy, repetition rate, and NA of the lens) strongly suggests the important role of thermal effects due to average heat deposition into the processed volume. Although the peak intensity of every individual pulse in our case $(\sim 5 \mathrm{MW})$ is much higher than a Kerr self-focusing power threshold of 
$\mathrm{Si}(\sim 22 \mathrm{~kW}$ estimated from [26]), it is evident that every single pulse, if it is sent with a low repetition rate, does not perform the modification. It is also consistent with previous studies, where a single femtosecond pulse has failed to create any subsurface modification even, with $90 \mu \mathrm{J}$ of pulse energy [20].

The laser-written waveguide structures were first analyzed with quantitative shadowgraphy [27] to identify the refractive index change with respect to the unmodified Si crystal. In order to obtain the shadow images of the fabricated structures (Fig. 2), we used the setup described in Fig. 1. However, in this case, the pump arm was blocked, and the probe arm was used as a light source. We observe that the modified section is almost a pure phase object, becoming invisible if the object is placed exactly in the focal plane of the camera objective. By translating the object $200 \mu \mathrm{m}$ from the focal plane, in the direction closer to the camera [Fig. 2(a)], as well as $200 \mu \mathrm{m}$ away from the camera [Fig. 2(b)], we observe a characteristic intensity contrast change in the central part of modified area. This behavior indicates a positive refractive index change $(\Delta n>0)$ for the laser-modified area, compared to the crystal matrix [26].

We applied inverse Abel transform to phase images of waveguides, which are computed from the corresponding shadow images [Figs. 2(a) and 2(b)] using the transport-ofintensity equation [27]. The calculated refractive index profiles
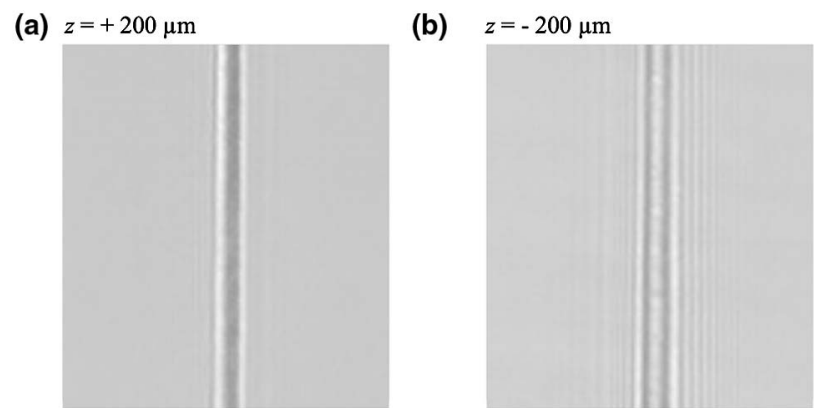

(c)

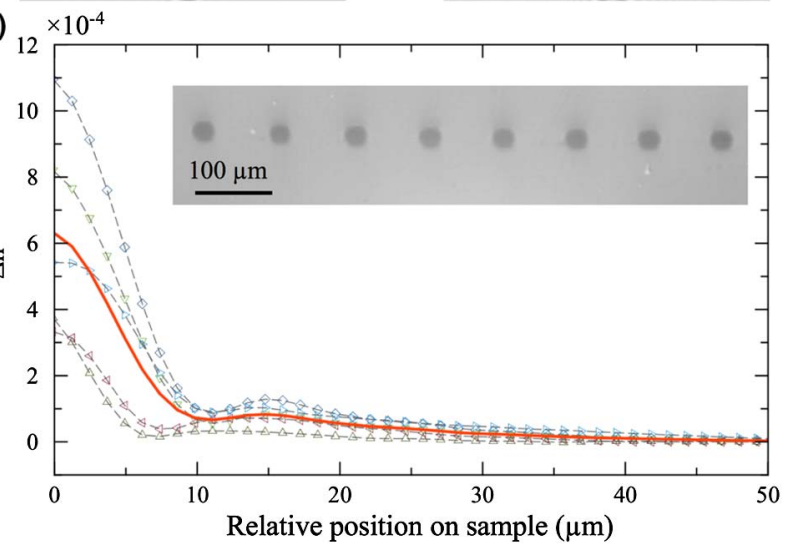

Fig. 2. Quantitative shadowgraphy of subsurface waveguides. (a) Shadow image of a waveguide obtained when the object is $200 \mu \mathrm{m}$ translated from the focal plane toward the camera. (b) Shadow image of the same waveguide in (a), obtained when the object is $200 \mu \mathrm{m}$ translated from the focal plane, away from the camera. (c) Refractive index profiles from five different waveguides, obtained by the inverse Abel transform method (black dashed lines). The red solid line shows the refractive index profile obtained by averaging five index profiles. The waveguides are fabricated with the same parameters. Inset: cross-sectional view of an array of waveguides, recorded with infrared transmission microscopy. are shown in Fig. 2(c). In order to reduce errors that may arise from the well-known sensitivity of this method to measurement errors, we reconstructed the refractive index profiles from five different waveguides fabricated with the same processing parameters, and averaged the obtained results [Fig. 2(c)]. These measurements yield a refractive index change of about $6 \times 10^{-4}$ at the center of the structures with respect to unmodified Si.

An array of $5.5 \mathrm{~mm}$ long, $100 \mu \mathrm{m}$ separated subsurface waveguides were written in Si for testing [inset of Fig. 2(c)]. Then, collimated light from a $10 \mathrm{~mW} \mathrm{cw}$ laser operating at $1.5 \mu \mathrm{m}$ was coupled into the waveguides with a lens of $\mathrm{NA}=0.2$ [Fig. 3(a)]. The polarization of the laser was the same as the polarization of the pump beam. As a control experiment, the beam was also focused in Si with the same lens at a location where there was no waveguide. The output light was recorded with an InGaAs camera, from a screen at a distance of $4 \mathrm{~cm}$, corresponding to the far-field intensity distributions for both the control beam [part i of Fig. 3(b)] and the output beam from a waveguide [part ii of Fig. 3(b)]. The dark shadows seen in the far-field waveguide image are due to neighboring waveguides, whereas the white halo around the main peak is attributed to residual uncoupled light. A representative near-field image at the exit port of a waveguide [part iii of Fig. 3(b)] demonstrates that the guided laser light is confined to a $\approx 20 \mu \mathrm{m}$ spot diameter. The intensity profiles obtained along the vertical symmetry axes of the far-field images are shown in parts $\mathrm{i}$ and ii of Fig. 3(b); the corresponding Gaussian fits are shown in Fig. 3(c). We calculated the NA of the decoupled light from the intensities given in Fig. 3(c). (The width is taken at $1 / e^{2}$ of the intensity profile.) For the unmodified area, the $\mathrm{NA}$ is found to be 0.19 , which is about the same as the NA of the focusing lens. The NA of the waveguide, in contrast, is calculated to be 0.05 . Assuming a step-index profile, this corresponds to a refractive index difference of $3.5 \times 10^{-4}$, which is in

(a)

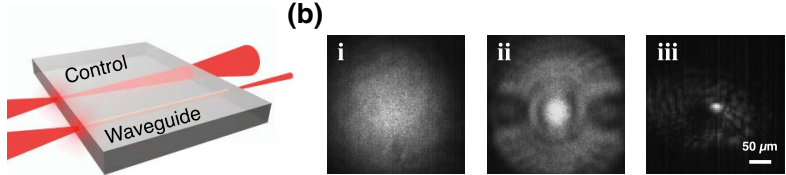

(c)

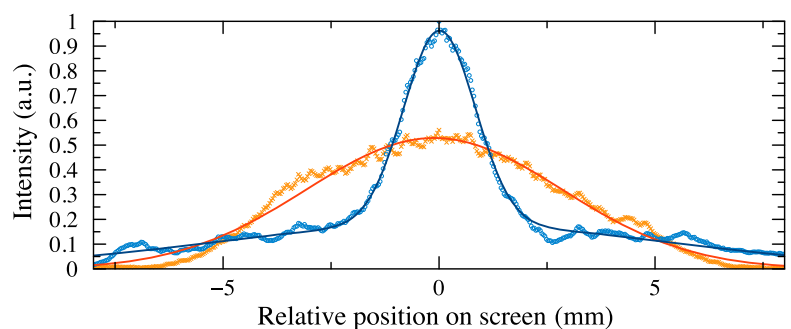

Fig. 3. Optical waveguides inside Si. (a) Schematic illustrates direct coupling of $1.5 \mu \mathrm{m} \mathrm{cw}$ laser light into a subsurface waveguide and the corresponding control experiment. (b) i, far-field image of $1.5 \mu \mathrm{m}$ laser light after passing through $\mathrm{Si}$ without waveguide. ii, far-field image of a $1.5 \mu \mathrm{m}$ laser light exiting from the output port of a subsurface waveguide. iii, near-field image of a $1.5 \mu \mathrm{m}$ laser light exiting from the output port of a subsurface waveguide. (c) Intensity profiles along the vertical axes of the far-field images shown in parts i, ii, and the corresponding numerical fits. The blue (solid) curve is a double Gaussian fit to the intensity profile data (blue circles) obtained from the waveguide exit. The red curve is a Gaussian fit to the data (orange crosses) from the control measurement. 
good agreement with the result obtained from the quantitative shadowgraphy method.

For waveguide loss characterization, we compared the output power from the unmodified Si [part i in Fig. 3(b)] and the power in the central maximum of the waveguide output in part ii in Fig. 3(b). Using $16 \mathrm{~mW}$ of incident laser power, the output power from the unmodified $\mathrm{Si}$ is measured to be $6.5 \mathrm{~mW}$. Here, the losses are mainly due to multiple reflections from the surfaces of the wafer. Similarly, the waveguide output power is measured as $0.45 \mathrm{~mW}$ [part ii in Fig. 3(b)], corresponding to $7.1 \%$ of the laser power from the unmodified $\mathrm{Si}$. With the given lens NA, the estimated coupling efficiency is $\approx 7-8 \%$, which shows that the actual loss of the waveguide is negligible, compared to the reflection and coupling losses.

In summary, we demonstrated optical waveguides written deep inside $\mathrm{Si}$ with a $1.5 \mu \mathrm{m}$ femtosecond laser. To the best of our knowledge, this is the first report of $3 \mathrm{D}$ controllable subsurface Si modification with femtosecond pulses, as well as the first demonstration of optical waveguides written with ultrafast laser pulses in Si. From the characterization of waveguides by optical shadowgraphy and direct light coupling, we measured the refractive index difference between the waveguide and unmodified crystal as $6 \times 10^{-4}$ and $3.5 \times 10^{-4}$, respectively. The waveguide diameter was measured to be $20 \mu \mathrm{m}$. With this fabrication method, one can create multi-level arrays of functional waveguides in Si. We anticipate that with future optimization of the laser and scanning parameters, it may be possible to create additional in-chip optical elements, with refractive index modulation control and various architectures. We believe this new laser-writing method will find use in 3D integrated optics, Si photonics, and optical chip-to-chip communications.

Funding. European Union's H2020 Marie SkłodowskaCurie Actions (MSCA) (660769); H2020 European Research Council (ERC) (NLL-617521); Türkiye Bilimsel ve Teknolojik Arastirma Kurumu (TÜBITAK) (114F256).

Acknowledgment. We thank Tahir Çolakoğlu of METU, Ankara, Turkey, and Murat Güre and Ergun Karaman of Bilkent University, Ankara, Turkey, for technical support.

\section{REFERENCES}

1. R. W. Keyes and J. A. Armstrong, Appl. Opt. 8, 2549 (1969).
2. D. A. B. Miller, Appl. Opt. 49, F59 (2010).

3. A. Sugama, K. Kawaguchi, M. Nishizawa, H. Muranaka, and Y. Arakawa, Opt. Express 21, 24231 (2013).

4. D. A. B. Miller, Proc. IEEE 97, 1166 (2009).

5. N. Sherwood-Droz and M. Lipson, Opt. Express 19, 17758 (2011).

6. G. Reed, Silicon Photonics (Wiley, 2008), pp. 80-81.

7. R. R. Gattass and E. Mazur, Nat. Photonics 2, 219 (2008).

8. B. C. Stuart, M. D. Feit, S. Herman, A. M. Rubenchik, B. W. Shore, and M. D. Perry, Phys. Rev. B 53, 1749 (1996).

9. C. W. Carr, H. B. Radousky, A. M. Rubenchik, M. D. Feit, and S. G. Demos, Phys. Rev. Lett. 92, 87401 (2004).

10. K. M. Davis, K. Miura, N. Sugimoto, and K. Hirao, Opt. Lett. 21, 1729 (1996).

11. M. Bazzan and C. Sada, Appl. Phys. Rev. 2, 040603 (2015).

12. A. Stone, H. Jain, V. Dierolf, M. Sakakura, Y. Shimotsuma, K. Miura, K. Hirao, J. Lapointe, and R. Kashyap, Sci. Rep. 5, 10391 (2015).

13. M. Pospiech, M. Emons, B. Väckenstedt, G. Palmer, and U. Morgner, Opt. Express 18, 6994 (2010).

14. I. Blonskyi, V. Kadan, O. Shpotyuk, M. Iovu, P. Korenyuk, and I. Dmitruk, Appl. Phys. B 104, 951 (2011).

15. F. Chen and J. R. V. de Aldana, Laser Photon. Rev. 8, 251 (2014).

16. G. Corrielli, A. Crespi, R. Geremia, R. Ramponi, L. Sansoni, A. Santinelli, P. Mataloni, F. Sciarrino, and R. Osellame, Nat. Commun. 5, 4249 (2014).

17. G. D. Marshall, A. Politi, J. C. F. Matthews, P. Dekker, M. Ams, M. J. Withford, and J. L. O'Brien, Opt. Express 17, 12546 (2009).

18. A. Nejadmalayeri, P. Herman, J. Burghoff, M. Will, S. Nolte, and A. Tunnermann, Opt. Lett. 30, 964 (2005).

19. D. Grojo, A. Mouskeftaras, P. Delaporte, and S. Lei, J. Appl. Phys. 117, 153105 (2015)

20. V. V. Kononenko, V. V. Konov, and E. M. Dianov, Opt. Lett. 37, 3369 (2012).

21. I. A. Pavlov, E. Dulgergil, E. Ilbey, and F. Ö. Ilday, Conference on Lasers and Electro-Optics (Optical Society of America, 2012), paper CTu2M.5.

22. O. Tokel, A. Turnali, G. Makey, P. Elahi, S. Ilday, T. Colakoglu, E. Ergecen, Ö. Yavuz, R. Hübner, M. Z. Borra, I. Pavlov, A. Bek, R. Turan, S. Tozburun, and F. O. Ilday, "In-chip microstructures and photonic devices fabricated by nonlinear laser lithography deep inside silicon," arXiv:1409.2827v3 [physics.optics] (2017).

23. M. Chambonneau, Q. Li, M. Chanal, N. Sanner, and D. Grojo, Opt. Lett. 41, 4875 (2016).

24. C. Kerse, H. Kalaycioglu, P. Elahi, B. Cetin, D. K. Kesim, Ö. Akçaalan, S. Yavas, M. D. Asik, B. Öktem, H. Hoogland, R. Holzwarth, and F. Ö. Ilday, Nature 537, 84 (2016).

25. V. Kadan, I. Blonskyy, I. Dmytruk, P. Korenyuk, I. Pavlov, V. Puzikov, E. Kryvonosov, and L. Lytvynov, Proc. SPIE 6726, 67260F (2007).

26. A. Mysyrowicz, A. Couairon, and U. Keller, New J. Phys. 10, 025023 (2008).

27. A. Gopal, S. Minardi, and M. Tatarakis, Opt. Lett. 32, 1238 (2007). 